\title{
An Elliptic Singular System with Nonlocal Boundary Conditions
}

\author{
Pablo Amster and Manuel Maurette
}

\begin{abstract}
We study the existence of solutions for the nonlinear elliptic system $\Delta u+g(u)=f(x)$, where $g \in C\left(\mathbb{R}^{N} \backslash S, \mathbb{R}^{N}\right)$ and $S$ is a bounded set of singularities. Using topological degree methods, we prove existence results. We analyze in particular the case in which $S=\{0\}$ and the isolated singularity is of a repulsive nature, by approximating problems and prove that if an appropriate Nirenberg type condition holds then the problem has a solution.
\end{abstract}

Keywords: singularities; elliptic system; nonlocal conditions; topological degree.

2010 MSC: 35D99,35J66.

\section{Introduction}

Let $\Omega \subset \mathbb{R}^{d}$ an open, connected and bounded set, we study the following Elliptic System:

$$
\left\{\begin{array}{cc}
\Delta u+g(u) & =f(x) \quad \text { in } \Omega \\
u & =C \quad \text { on } \partial \Omega \\
\int_{\partial \Omega} \frac{\partial u}{\partial \nu} d S & =0
\end{array}\right.
$$

With $C \in \mathbb{R}^{N}$ an unknown constant vector, $f: \bar{\Omega} \rightarrow \mathbb{R}^{N}$ continuous such that $\bar{f}=\frac{1}{|\Omega|} \int_{\Omega} f(x) d x=0$ and $g: \mathbb{R}^{N} \backslash S \rightarrow R^{N}$ continuous, with $S$ a bounded set, representing the singularities.

This kind of singularities where studied for the ODE $(d=1)$ periodic case in [13], [7] and in [8], where it is remarked that the difficulty relies in the case $\bar{f}=0$.

Berestycki and Brézis in [5] and Ortega in [11] studied this nonlocal conditions, that arise from certain problems in plasma physics. In particular, it originates from a model describing the equilibrium of a plasma confined in a toroidal cavity, called a Tokamak machine. A study of this problem can be found in the appendix of [16]. 
Note that when $d=1$ and $\Omega=(a, b)$ an open interval, the system reads:

$$
u^{\prime \prime}+g(u)=p(t), \quad t \in(a, b)
$$

In this framework, nonlocal conditions represent:

$$
u=C \text { on } \partial \Omega \quad \Rightarrow \quad u(a)=u(b) ; \quad \int_{\partial \Omega} \frac{\partial u}{\partial \nu} d S=0 \quad \Rightarrow \quad u^{\prime}(a)=u^{\prime}(b) .
$$

Hence, this nonlocal condition can be seen as a generalization of a periodic-type condition. In this case, the authors, using topological degree methods, proved in [3] that when the nonlinearity $g: \mathbb{R}^{N} \backslash\{0\} \rightarrow \mathbb{R}^{N}$ is continuous, repulsive at the origin and with some boundness condition at infinity, if an appropriate Nirenberg type condition holds then either the problem has a classical solution, or else there exists a family of solutions of perturbated problems that converges uniformly and weakly in $H^{1}$ to some limit function $u$. Furthermore, under appropriate conditions it was shown that $u$ is in fact a classical solution. From now on we assume $d>1$.

This work will attack two essentially different problems. In the next section we will focus our attention on the hypothesis over $g$ and not ask anything on $S$. The idea is to use degree theory techniques on an appropriate open set in $C\left(\bar{\Omega}, \mathbb{R}^{N}\right)$. The hypothesis that we will work with is of a geometric nature. We will ask the point 0 to be separated from the convex hull of the image of $g$ in a ball around points in the boundary of a certain set $D \subset \mathbb{R}^{N} \backslash S$ :

$\left(D_{1}\right)$ For all $v \in \partial D, 0 \notin \operatorname{co}\left(g\left(\overline{B_{r}}(v)\right)\right)$

Of course we will also need a degree condition:

$\left(D_{2}\right) \operatorname{deg}(g, D, 0) \neq 0$,

Condition $\left(D_{1}\right)$ was introduced by Ruiz and Ward in [12] and followed in [2] by Amster and Clapp. It generalizes in a way the classical Nirenberg type conditions [10].

In our problem an apriori bound for $\|\nabla u\|_{\infty} \leq K$ will be found down in Section 2 (see (5) and a constant $d_{\Omega}$ that measures the convexity of $\Omega$ will also be involved in the hypothesis. This is a big difference from the $d=1$ case, where the domain is an interval and this kind of condition is not asked. Condition (B) is a boundary condition at $|u| \rightarrow \infty$. The main result in Section 2 is:

Theorem 1.1 Let $g \in C\left(\mathbb{R}^{N} \backslash S, \mathbb{R}^{N}\right)$ satisfying $(B)$ condition and $f \in$ $C\left(\bar{\Omega}, \mathbb{R}^{N}\right)$ such that $\bar{f}=0$. Let $\mathcal{C}$ be a compact neighborhood of $S$. Let $r>d_{\Omega} K$, if there exists a domain $D \subset \mathbb{R}^{N} \backslash\left(\mathcal{C}+\overline{B_{r}(0)}\right)$ such that $\left(D_{1}\right)$ and $\left(D_{2}\right)$ hold, then (1) has at least one solution $u$ satisfying $\bar{u} \in D$ and $\|u-\bar{u}\|_{\infty}<r$. 
In Section 3 we study the case when $S=\{0\}$ (namely any point in $\mathbb{R}^{N}$ by translation). In this case, we will focus our attention on the way $g$ behaves near the singular point. In fact, we will only consider the case in which the singularity is of a (strongly)repulsive type, that is :

(Rep) there exists $c>0$ such that $\langle g(u), u\rangle<0$ for $0<|u|<c$,

(SSR) there exists a sequence $r_{n} \searrow 0$ such that

$$
\sup _{|u|=r_{n}}\left\langle g(u), \frac{u}{|u|}\right\rangle \rightarrow-\infty \quad \text { as } n \rightarrow \infty .
$$

The first one is the classic repulsiveness condition. We called the second one the sequentially strongly repulsive condition.

In this case we will perturbate the problems, show results for the nonsingular case by topological degree techniques and study the limit problem.

First we introduce the approximated problems. Given $n \in \mathbb{N}$ :

$$
\Delta u+g_{n}(u)=f(x) \quad \text { in } \Omega
$$

satisfying the same boundary conditions. Here $g_{n}$ is a continuous (nonsingular) perturbation of $g$. For instance, let us show an example of such approximations:

$$
g_{n}(u)=\left\{\begin{array}{cl}
g(u) & |u| \geq \varepsilon_{n} \\
\rho_{n}(|u|) g\left(\varepsilon_{n} \frac{u}{|u|}\right) & 0<|u|<\varepsilon_{n} \\
0 & u=0
\end{array}\right.
$$

where $\varepsilon_{m} \rightarrow 0, \rho_{n}:\left[0, \varepsilon_{n}\right] \rightarrow[0,+\infty)$ is continuous and satisfies $\rho_{n}(0)=$ $0, \rho_{n}\left(\varepsilon_{n}\right)=1$. Note that here $g_{n} \rightarrow g$ uniformly when $n \rightarrow \infty$. When this is the case, we shall call these approximations, admissible ones.

The conditions over $g$ are of a similar kind as in section 2, but we will strength them to overcome the repulsiveness condition. They are other kind of generalization of the classical Nirenberg type conditions [10]. They have been developed by one of the authors in [1] and have been studied also in [3] for a system of singular periodic ordinary differential equations. The idea is to weaken the limit condition by taking a finite covering of the sphere and asking that over each set $U_{j}$ of the covering, there is a direction $w_{j}$ such that the product $\left\langle g\left(s U_{j}\right), w_{j}\right\rangle<0$ for $s$ large enough, i.e. $g\left(s U_{j}\right)$ is separated with a hyperplane from the origin by the Hahn Banach Theorem :

$\left(P_{1}\right)$ There exists a family $\mathcal{F}=\left\{\left(U_{j}, w_{j}\right)\right\}_{j=1, \ldots, J}$ where $\left\{U_{j}\right\}_{j=1, \ldots, J}$ is an open cover of $S^{N-1}$ and $w_{j} \in S^{N-1}$, such that for some $R_{j}>0$ and $j=1, \ldots, K$ :

$$
\left\langle g(r u), w_{j}\right\rangle<0 \quad \forall r>R_{j} \quad \forall u \in U_{j} .
$$


We will also consider a different kind of degree condition for this case as the repulsiveness condition $(S S R)$ will assure us that the degree near the origin will be $\left(-1^{N}\right)$. The condition we will look for is:

$\left(P_{2}\right)$ There exists a $R_{0}>0$ such that $\operatorname{deg}\left(g, B_{R}, 0\right) \neq(-1)^{N}$ for $r \geq R_{0}$.

We now state the main result of this section:

Theorem 1.2 Let $\Omega \subset \mathbb{R}^{d}$ a bounded $C^{2}$ domain. Let $g: \mathbb{R}^{N} \backslash\{0\} \rightarrow \mathbb{R}^{N}$ be continuous satisfying $(B),(\operatorname{Rep}),(S S R)$ and $f \in C\left(\bar{\Omega}, \mathbb{R}^{N}\right)$ with $\bar{f}=0$. Suppose that $\left(P_{1}\right)$ and $\left(P_{2}\right)$ hold. Let $\left\{g_{n}\right\}$ be as in (3) and $\left\{u_{n}\right\}_{n}$ solutions of (2). Then, there exists $u$ a generalized solution of (1).

The concept o generalized solution will be explained in detail later on, but in a few words it will mean that it satisfies a distributional problem associated to the original one. Of course we will also show that if $u$ is not 0 in $\Omega$ the generalized solution will indeed be a classical solution.

It is worth noticing that in this case we ask for more general conditions that those asked in section 2. Here, the restrictive condition is placed in the behavior singularity instead of in the condition over $S$.

Remark 1.3 We work with this boundary condition because of the interesting generalization of the periodic boundary condition in ODEs, but all the results proved in this work are also valid for Neumann boundary conditions. The crucial fact involved here is the fact that the problem is resonant. The Kernel of the Laplacian with the nonlocal boundary conditions (as well as with the Neumann conditions) has finite dimension. Hence, Mawhin's Continuation Theory [9] can be applied.

\section{The general case}

We will first look for a priori bounds of solutions of problem (1). After that, this bounds will allow us to use Continuation Theory to prove that the problem can be deformed to a more simple problem. Degree theory is used to prove existence of solution of this simpler problem.

Consider problem (1) with $g$ satisfying the following boundness condition:

(B) $\quad \lim \sup _{|u| \rightarrow \infty}|g(u)|<\infty$.

Given $\mathcal{C}$ a compact neighborhood of $S$, there exists $M=M_{\mathcal{C}}$ such that

$$
|g(u)| \leq M \quad u \in \mathbb{R}^{N} \backslash \mathcal{C} .
$$

Now, if $u$ is a solution of the system, supposing that $u(x) \in \mathbb{R}^{N} \backslash \mathcal{C}, \forall x$ we have 


$$
\|\nabla u\|_{\infty} \leq k\|\Delta u\|_{\infty}=k\|f-g(u)\|_{\infty} \leq k\left(\|f\|_{\infty}+M\right)=K
$$

Using the fact that $\|u-\bar{u}\|_{\infty} \leq \operatorname{diam}_{d}(\Omega)\|\nabla u\|_{\infty}$, with the distance $d(x, y)=\inf \{\operatorname{long}(\gamma): \gamma$ a piecewise smooth curve in $\Omega$ conn. $x$ and $y\}$, we have, calling $d_{\Omega}=\operatorname{diam}_{d}(\Omega)$ :

$$
\|u\|_{\infty} \leq\|u-\bar{u}\|_{\infty}+|\bar{u}| \leq d_{\Omega}\|\nabla u\|_{\infty}+|\bar{u}| \leq d_{\Omega} K+|\bar{u}| .
$$

Finally, taking a radius $r>d_{\Omega} K$, we can assure that if $u$ is a solution of the problem, then $\|\nabla u\|_{\infty}$ is bounded and $\|u-\bar{u}\|_{\infty}<r$. We are in condition to prove Theorem (1.1).

Proof of Theorem 1.1:

The proof relies in the Continuation Theory of Mawhin [9] applied to the set $U=\left\{u \in C\left(\bar{\Omega}, \mathbb{R}^{N}\right):\|u-\bar{u}\|_{\infty}<r, \bar{u} \in D\right\}$. Setting $\lambda \in(0,1]$, we study the problems

$$
\left\{\begin{array}{ccc}
\Delta u+\lambda g(u) & =\lambda f(x) & \text { in } \Omega \\
u & =C & \text { on } \partial \Omega \\
\int_{\partial \Omega} \frac{\partial u}{\partial \nu} d S & =0 &
\end{array}\right.
$$

It is easy to see that the exact same computations done before can be applied to this problems. If $u \in U$ is a solution of (6), $\|u-\bar{u}\|_{\infty}<r$ and $\|\nabla u\|_{\infty} \leq K$. Suppose now that $u \in \partial U$. There are two cases:

i) $\|u-\bar{u}\|_{\infty}=r$ : This case is leads to a trivial contradiction.

ii) $\bar{u} \in \partial D$. In this case, using hypothesis $\left(D_{1}\right)$, as the set $g(u(\bar{\Omega}))$ is compact we know because of the Hahn-Banach Theorem of the existence of a $w \in S^{N-1}$ such that $\langle g(v), w\rangle>0$ for all $v \in \operatorname{Im}(u) \subset \subset B_{r}(\bar{u})$, hence, writing $u=u-\bar{u}+\bar{u} \in B_{r}(\bar{u})$, as $\|u-\bar{u}\|_{\infty}<r$ we have that $\langle g(u), w\rangle>0$. On the other hand, a simple computation directly from (1) shows that $\int_{\Omega} g(u(x)) d x=0$, arriving at a contradiction.

Finally we have to check the degree condition, $\operatorname{deg}_{L S}\left(I-T_{0}, U, 0\right) \neq 0$, where $T_{\lambda} u=\bar{u}-\overline{(f-g(u))}+\lambda L^{-1}((f-g(u))-\overline{(f-g(u))})$, with $L^{-1}$ such that if $L^{-1} \varphi=u$ with $u$ the only solution of the problem:

$$
\left\{\begin{array}{ccc}
\Delta u & =\varphi \quad \text { in } \Omega \\
u & =C \quad \text { on } \partial \Omega \\
\int_{\partial \Omega} \frac{\partial u}{\partial \nu} d S & =0 \\
\frac{u}{u} & =0
\end{array}\right.
$$

Here, $\Delta$ is an operator from $C\left(\bar{\Omega}, \mathbb{R}^{N}\right) \cap B . C$., where B.C. is the set of functions that satisfy the boundary conditions. Restricted to the subspace where $\bar{u}=0$ this operator, named $L$ is invertible.

Now, as $\bar{f}=0$, we have: 


$$
T_{\lambda} u=\bar{u}+\overline{g(u)}+\lambda L^{-1}((f-g(u))+\overline{g(u)}) .
$$

Hence, we can now prove the last statement:

$$
\begin{gathered}
\operatorname{deg}_{L S}\left(I-T_{0}, U, 0\right)=\operatorname{deg}\left(\left.\left(I-T_{0}\right)\right|_{U \cap \mathbb{R}^{N}}, U \cap \mathbb{R}^{N}, 0\right)= \\
=\operatorname{deg}\left(-g, U \cap \mathbb{R}^{N}, 0\right)=\operatorname{deg}(-g, D, 0)=(-1)^{N} \operatorname{deg}(g, D, 0) \neq 0 .
\end{gathered}
$$

The last equalities are due to the fact that if $u \in U \cap \mathbb{R}^{N}$, then on one hand $u=\bar{u}$, and on the other, $\bar{u} \in D$, so $u \in D$ (and vice versa) and condition $\left(D_{2}\right)$.

Remark 2.1 In this framework, the continuous case, that is, the case where $g$ has no singularities can be just seen as a particular case of the above, when $S=\emptyset$. Here, $K=k\|f\|_{\infty}, r>d_{\Omega} K$ and $\mathcal{C}$ is also the empty set, so the hypothesis would be: $\exists D$, such that $\left(D_{1}\right)$ and $\left(D_{2}\right)$ hold, a result similar of that showed in [2], that generalizes the more classical condition where $D=B_{R}(0)$.

Remark 2.2 Condition $(B)$ can be weakened and ask $g$ to be sub-linear:

$$
\lim _{|u| \rightarrow \infty} \frac{|g(u)|}{|u|}<\infty
$$

The result is obtained, but the a-priori bounds (for example the computation of $K(5)$ are more technical.

Let us show an example that illustrates the possibility of getting multiple solutions. We call from now on $B_{h}=B_{h}(0)=\left\{u \in \mathbb{R}^{N}:|u|<h\right\}$.

Example 2.3 Let $A: \mathbb{R}^{N} \rightarrow \mathbb{R}^{N}$ a continuous and bounded function, $\|A\|_{\infty} \leq a, b>0$ and $\Omega \subset \mathbb{R}^{d}$. Consider the case where $g(u)=\frac{A(u)}{|u|(b-|u|)}$, so that $S=\{0\} \cup \partial B_{b}$. Let $\eta>0$ and take the following compact set:

$$
\mathcal{C}=\mathcal{C}_{1} \cup \mathcal{C}_{2}=\overline{B_{\eta}} \cup\left(B_{b+\eta} \backslash B_{b-\eta}\right) .
$$

Hence, $\mathbb{R}^{N} \backslash \mathcal{C}=\{\eta<|u|<b-\eta\} \cup\{|u|>b+\eta\}$.

The bound of the gradient, previously called $K$, in this case is:

$$
\|\nabla u\|_{\infty} \leq K=k\left(\|f\|_{\infty}+\frac{\|A\|_{\infty}}{\eta(b+\eta)}\right)
$$

Note that for this case, the radius would have to be

$$
r \geq d_{\Omega} k\left(\|f\|_{\infty}+\frac{a}{\eta(b+\eta)}\right)
$$


Note that for instance, if such $r$ also satisfies $b>2(r+\eta)$, it would be possible the existence of two disjoints sets $D^{1}, D^{2} \subset \mathbb{R}^{N} \backslash\left(\mathcal{C}+B_{r}\right)$ such that:

$$
D^{1} \subset B_{b-\eta-r} \backslash B_{\eta+r}, \quad D^{2} \subset \mathbb{R}^{N} \backslash B_{b+\eta+r}
$$

that would lead to two especially different solutions of the system, $u_{1}$ and $u_{2}$ with $\overline{u_{1}} \in D^{1}$ and $\overline{u_{2}} \in D^{2}$ respectively.

For this to happen we would have to be able to find an $r$ such that

$$
R k\left(\|f\|_{\infty}+\frac{a}{\eta(b+\eta)}\right)<r ; \quad r<b-\eta ; \quad r<\frac{b}{2}-\eta
$$

Condition $\left(D_{1}\right)$ also requires that $\eta+2 r<b-\eta-2 r$, the condition for $b$ is then:

$$
b>4 r+2 \eta .
$$

Let us show an explicit $g$ that would allow to have two solutions.

Let $\tilde{g}: \mathbb{R}^{+} \backslash\{0, b\} \rightarrow \mathbb{R}$ the following function

$$
\tilde{g}(x)=\frac{\left(x-x_{1}\right)\left(x-x_{2}\right)}{x(x-b)}
$$

Suppose also that $x_{1} \in(\eta+2 r, b-\eta-2 r)$ and $x_{2}>b+\eta+2 r$. The following figure describes the situation:

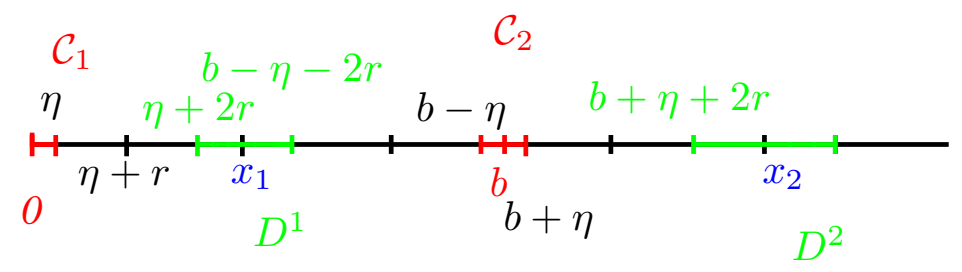

Let now be $g: \mathbb{R}^{N} \backslash S \rightarrow \mathbb{R}^{N}$

$$
g(u)=\tilde{g}(|u|) u=\frac{\left(|u|-x_{1}\right)\left(|u|-x_{2}\right) u}{|u|(|u|-b)}
$$

It is clear that for this $g$, all the conditions are satisfied for $D^{1}$ and $D^{2}$ independent of each other. Hence Theorem 1.1 says that there exist $u^{1}, u^{2}$ classical solutions of problem (1) and $\overline{u^{i}} \in D^{i}$, for $i=1,2$, this means that they are indeed different.

Remark 2.4 This example shows that it is crucial the distance between the conected components of the set $S$. If they are close, it would be impossible to find a suitable $r$ to use Theorem 1.1.

Note that a similar analysis can be made if the set $S$ is a disjoint union of sets $S_{i}$ such that the distance between this sets is sufficiently large as 
for to have sets $D^{i}$ included in the complement of the union of compact neighbourhoods, and solutions $u^{i}$ such that $\bar{D}^{i}$.

\section{The case $S=\{0\}$}

Let us define the concept of generalized solution previously mentioned. Let $u_{n}$ be weak solution for (2) such that $u_{n} \rightarrow u$ weakly in $H^{1}$ and $\left\|\nabla u_{n}\right\|_{L^{2}} \leq$ $c>0, u_{n} \in H=H_{0}^{1}+\mathbb{R}^{N}$ and it satisfies

$$
\int_{\Omega} \Delta u_{n} \varphi+\int_{\Omega} g_{n}\left(u_{n}\right) \varphi=\int_{\Omega} f \varphi \quad \forall \varphi \in H
$$

Because the first term on the left, and the term on the right have a limit, we can define the following operator: $A: H \rightarrow \mathbb{R}^{N}$ as:

$$
A \varphi=\lim _{n \rightarrow \infty} \int_{\Omega} g_{n}\left(u_{n}\right) \varphi
$$

First, it is easy to prove that the functional $A$ is continuous and bounded in $H$, so $A \in H^{-1}$ :

$$
A \varphi=\int_{\Omega} f \varphi d x+\sum_{j=1} \nabla u^{j} \nabla \varphi^{j} d x
$$

Note that we can see the functional $A$ as a pair $(f, \nabla u) \in H^{-1}$ such that it operates:

$$
A[\varphi]=(f, \nabla u)[\varphi] .
$$

We shall then define the following operator $\hat{g}: H \rightarrow H^{-1}$ :

$$
\hat{g}(u):=(f, \nabla u) ; \quad \hat{g}(u)[\phi]:=(f, \nabla u)[\varphi]=A[\phi] .
$$

Note that this definition makes in fact generalizes $g$. If $u \neq 0$ never, then, taking $\varphi \in H$

$$
\hat{g}(u)[\varphi]=A[\varphi]=\lim _{n \rightarrow \infty} \int_{\Omega} g_{n}\left(u_{n}\right) \varphi d x=\int_{\Omega} g(u) \varphi d x
$$

So one has that $\Delta u+\hat{g}(u)=f$, but on the other hand, as $u$ was never zero, then the problem is not singular and it satisfies $\Delta u+g(u)=f$. So this definition indeed generalizes in a distributional meaning the concept of solution. This is what we call a generalized solution:

Definition 3.1 A function $u \in H$ is said to be a generalized solution of (1) if for some admissible choice of $g_{n}$ there exist sequences $\varepsilon_{n} \rightarrow 0$ and $\left\{u_{n}\right\}$ solutions of (2). such that $u_{n} \rightarrow u$ weakly in $H^{1}$ and $u$ is a solution of the problem 


$$
\Delta u+\hat{g}(u)=f
$$

with $\hat{g}$ as in (8), in a distribution sense.

The idea is to use the above mentioned perturbed problems, get solutions of those via results for the continuous case (using that $S=\emptyset$ ), and obtain a sequence $u_{n}$ of solutions of these problems. Finally we will look for uniform bounds for this approximated solutions to have some limit function $u$ and prove that it is indeed a generalized solution of (1).

Let us state a result for the nonsingular case

Theorem 3.2 Let $\Omega \subset \mathbb{R}^{d}$ a bounded $C^{2}$ domain, and let $g \in C\left(\mathbb{R}^{N}, \mathbb{R}^{N}\right)$ such that $(B)$ hold, and $f \in C\left(\bar{\Omega}, \mathbb{R}^{N}\right)$ with $\bar{f}=0$. Furthermore, if $\left(P_{1}\right)$ and $\left(D_{2}\right)$ hold with $D=\{\tilde{r}<|u|<R\}$ an anellus in $\mathbb{R}^{N}$ with $r$ sufficiently small and $R$ sufficiently large, then problem (1) admits a classical solution $u$.

Proof:

We will prove that condition $\left(P_{1}\right)$ imply $\left(D_{2}\right)$ in this context and directly apply Theorem 1.1 in the case $S=\emptyset$.

Taking $R_{0}=\max \left\{R_{j}: 1 \leq j \leq J\right\}+r$, with $r$ as in the Theorem 1.1, let us define $D=B_{R_{0}}(0)$.

Now, if $v \in \partial D$, then $v=R_{0} w$ with $w \in S^{N-1}$. As the $\left\{U_{j}\right\}$ cover the sphere, there exist $j_{0}$ such that $w \in U_{j_{0}}$ and, by $\left(P_{1}\right)$, as $R_{0} \geq R_{j}$ we know that $\langle g(v), 0\rangle<0$. And the same happens in a neighborhood of radius $r$, by construction of $R_{0}$, so:

$$
\langle g(u), 0\rangle<0 \quad \forall u \in B_{r}(v)
$$

Hence, $0 \notin c o\left(g\left(B_{r}(v)\right)\right)$ and condition $\left(D_{1}\right)$ is satisfied.

We now focus our attention to problem (2) with the $g_{n}$ as in (3). The following Lemma says that the solutions of the perturbated problems are uniformly bounded not only from above, but also from below:

Lemma 3.3 Let $g \in C\left(\mathbb{R}^{N} \backslash\{0\}, \mathbb{R}^{N}\right)$ satisfying (4) (Rep) and (SSR) and $f: \bar{\Omega} \rightarrow \mathbb{R}^{N}$ with $\bar{f}=0$. Let, for $n=1,2, \ldots, g_{n}$ be as in (3) and $u_{n}$ a solution of problem (2). There exists $n_{0} \in \mathbb{N}$ and $a \tilde{r}>0$ such that $\tilde{r}<\left\|u_{n}\right\|_{\infty} \forall n \geq n_{0}$. Moreover, $\tilde{r}$ does not depend on $f$.

Proof:

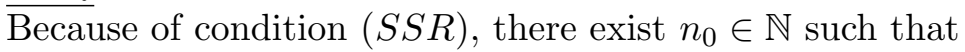

$$
\left\langle g(u), \frac{u}{|u|}\right\rangle+\|f\|_{L^{\infty}}<0 \text { for }|u|=r_{n_{0}} .
$$

Let us call $\tilde{r}:=r_{n_{0}}$ and see that if $\|u\|_{L^{\infty}}=\tilde{r}, u$ is not a solution of (6): 
If this was the case, then fix $x_{0}$ such that $\|u\|_{L^{\infty}}=\left|u\left(x_{0}\right)\right|=\tilde{r}$. Defining the auxiliary function $\phi(x):=\frac{|u(x)|^{2}}{2}$ let us see that we arrive to a contradiction.

If $x_{0} \in \Omega$, then

$$
\begin{gathered}
\Delta \phi\left(x_{0}\right)=\left|\nabla u\left(x_{0}\right)\right|^{2}+\left\langle u\left(x_{0}\right), \Delta u\left(x_{0}\right)\right\rangle \geq\left\langle u\left(x_{0}\right), f\left(x_{0}\right)-g\left(u\left(x_{0}\right)\right)\right\rangle= \\
=\lambda\left[\left\langle u\left(x_{0}\right), f\left(x_{0}\right)\right\rangle-\left|u\left(x_{0}\right)\right|\left\langle g\left(u\left(x_{0}\right)\right), \frac{u\left(x_{0}\right)}{\left|u\left(x_{0}\right)\right|}\right\rangle\right] \geq \\
\geq \tilde{r}\left[-\|f\|_{\infty}-\left\langle g\left(u\left(x_{0}\right)\right), \frac{u\left(x_{0}\right)}{\left|u\left(x_{0}\right)\right|}\right\rangle\right]>0,
\end{gathered}
$$

a contradiction, because $x_{0}$ would be a maximum of $\phi$. For the previous bound we used (9).

If $x_{0} \in \partial \Omega$, we have that $\tilde{r}$ has to be equal to $|C|$.

$$
\int_{\partial \Omega} \frac{\partial \phi}{\partial \nu} d S=\int_{\partial \Omega}\left\langle u, \frac{\partial u}{\partial \nu}\right\rangle d S=\int_{\partial \Omega}\left\langle C, \frac{\partial u}{\partial \nu}\right\rangle d S=\left\langle C, \int_{\partial \Omega} \frac{\partial u}{\partial \nu} d S\right\rangle=0 .
$$

Because of the continuity of $\phi$, for some $\delta>0$, and arguing as before, we have that $\Delta \phi>0$ in $B_{2 \delta}\left(x_{0}\right) \cap \Omega$.

From the standard regularity theory, it follows that $u \in C^{2}(\Omega) \cap C^{1}(\bar{\Omega})$. Moreover, we may consider a $C^{2}$ domain $\Omega_{0} \subset \Omega$ such that $B_{\delta} \cap \Omega \subset \Omega_{0} \subset$ $B_{2 \delta} \cap \Omega$; then $\phi\left(x_{0}\right)>\phi(x)$ for every $x \in \Omega_{0}$, and then from Hopf's Lemma we obtain

$$
\frac{\partial \phi}{\partial \nu}\left(x_{0}\right)>0
$$

As $u \equiv C$ in the boundary, then $|u(x)|=|C|=\tilde{r}$ also in the boundary. The same argument can be used for each element of $\partial \Omega$. This contradicts (10).

This same calculation can be made for every $0<r \leq \tilde{r}$, hence the lemma is proved.

We now would like to have uniform bounds from above also i.e $\left\|u_{n}\right\|_{\infty} \leq$ $R$ for $n$ big en0ugh. Recently, Arcoya et al. [4] and Boccardo-Orsina [6] used a well known Stampacchia result (see $[14,15]$ ) to obtain uniform bounds to approximated problems of a different nature. Before so, let us show that the $u_{n}$ are bounded in the $H^{1}$ norm.

Lemma 3.4 There exist a sequence $u_{n} \in H^{1}(\Omega)$ of solutions of the approximated problems with the proper $g_{n}$ such that $\left\|u_{n}\right\|_{H^{1}} \leq \mathcal{C}$. 
Proof:

$\overline{\text { As } \Delta} u_{n}+g_{n}\left(u_{n}\right)=f(x)$ in $\Omega$ and $u_{n} \equiv C_{n}$ on $\partial \Omega$ (note that each $u_{n}$ takes a different constant vector value $C_{n}$ on the boundary. Multiplying by $u_{n}-C_{n}$ and integrating in $\Omega$ :

$$
\int_{\Omega}\left\langle\Delta u_{n}+g_{n}\left(u_{n}\right), u_{n}-C_{n}\right\rangle d x=\int_{\Omega}\left\langle p, u_{n}-C_{n}\right\rangle d x
$$

Integrating by parts, the left hand side is equal to:

$$
-\int_{\Omega}\left|\nabla u_{n}\right|^{2} d x+\int_{\partial \Omega}\left\langle\frac{\partial u_{n}}{\partial \nu}, u_{n}-C_{n}\right\rangle d S+\int_{\Omega}\left\langle g_{n}\left(u_{n}\right), u_{n}-C_{n}\right\rangle d x
$$

Using that $u_{n} \equiv C$ on $\partial \Omega$, then $u_{n}-C_{n}=0$ on $\partial \Omega$. Hence,

$$
\left\|\nabla u_{n}\right\|_{L^{2}}^{2}=\int_{\Omega}\left\langle g_{n}\left(u_{n}\right), u_{n}-C_{n}\right\rangle d x-\int_{\Omega}\left\langle p, u_{n}-C_{n}\right\rangle d x
$$

Now, taking absolute value and using the Cauchy-Schwarz inequality, we get

$$
\left\|\nabla u_{n}\right\|_{L^{2}}^{2} \leq\left|\int_{\Omega}\left\langle g_{n}\left(u_{n}\right), u_{n}-C_{n}\right\rangle d x\right|+\|p\|_{L^{2}}\left\|u_{n}-C_{n}\right\|_{L^{2}}
$$

Let us study the first right side term, taking $c$ as in (Rep):

$$
\begin{aligned}
\left|\int_{\Omega}\left\langle g_{n}\left(u_{n}\right), u_{n}-C_{n}\right\rangle d x\right| \leq & \left|\int_{\left\{\left|u_{n}\right|<c\right\}}\left\langle g_{n}\left(u_{n}\right), u_{n}-C_{n}\right\rangle d x\right| \\
& +\left|\int_{\left\{\left|u_{n}\right| \geq c\right\}}\left\langle g_{n}\left(u_{n}\right), u_{n}-C_{n}\right\rangle d x\right|
\end{aligned}
$$

There is an $n_{0} \in \mathbb{N}$ such that $\frac{1}{n}<c$ for every $n \geq n_{o}$, and considering that $g_{n}\left(u_{n}\right)=g\left(u_{n}\right)$ if $\left|u_{n}\right|>c>\frac{1}{n}$ we have on one hand:

$$
\left|\int_{\left\{\left|u_{n}\right| \geq c\right\}}\left\langle g_{n}\left(u_{n}\right), u_{n}-C_{n}\right\rangle d x\right| \leq|\Omega| \sup _{|u|>c}|g(u)|^{2}\left\|u_{n}-C_{n}\right\|_{L^{2}}
$$

and on the other hand, the remaining term can be written as:

$$
\int_{\left\{\left|u_{n}\right|<c\right\}}\left\langle g_{n}\left(u_{n}\right), u_{n}\right\rangle d x-\int_{\left\{\left|u_{n}\right|<c\right\}}\left\langle g_{n}\left(u_{n}\right), C_{n}\right\rangle d x
$$

From (Rep) the first term is non-positive, moreover, as $\int_{\Omega} g_{n}\left(u_{n}\right) d x=0$, we deduce that $\int_{\left\{\left|u_{n}\right|<c\right\}} g_{n}\left(u_{n}\right) d x=-\int_{\left\{\left|u_{n}\right| \geq c\right\}} g_{n}\left(u_{n}\right) d x$. Hence

$$
\int_{\left\{\left|u_{n}\right|<c\right\}}\left\langle g_{n}\left(u_{n}\right), u_{n}-C_{n}\right\rangle d x \leq\left\langle C_{n}, \int_{\{|u| \geq c\}} g_{n}\left(u_{n}\right)\right\rangle d x
$$


So, we have, for this last term:

$$
\left|\int_{\left\{\left|u_{n}\right|<c\right\}}\left\langle g_{n}\left(u_{n}\right), u_{n}-C_{n}\right\rangle d x\right| \leq\left|C_{n}\right||\Omega| \sup _{|u|>c}|g(u)|
$$

Gathering all together:

$$
\left|\int_{\Omega}\left\langle g_{n}\left(u_{n}\right), u_{n}-C_{n}\right\rangle d x\right| \leq|\Omega| \sup _{|u|>c}|g(u)|^{2}\left\|u_{n}-C_{n}\right\|_{L^{2}}+C_{n}|| \Omega\left|\sup _{|u|>c}\right| g(u) \mid
$$

Thus,

$$
\left\|\nabla u_{n}\right\|_{L^{2}}^{2} \leq \mathcal{C}_{1}\left\|u_{n}-C_{n}\right\|_{L^{2}}+\mathcal{C}_{2}\left|C_{n}\right|
$$

for some constants $\mathcal{C}_{1}, \mathcal{C}_{2}$. Using Poincare inequality, we finally have that there exist some constant $\mathcal{C}$ big enough such that:

$$
\left\|\nabla u_{n}\right\|_{L^{2}} \leq \mathcal{C}\left|C_{n}\right|^{1 / 2}
$$

Note that we also have that $\left\|u_{n}-C_{n}\right\|_{L^{2}}^{2} \leq \mathcal{C}\left|C_{n}\right|$. One important consequence of the last bound is the following one:

$$
\left\|u_{n}-C_{n}\right\|_{H^{1}}^{2} \leq A+B\left|C_{n}\right| \quad \text { for some } A, B>0
$$

If we now suppose that $\left|C_{n}\right|$ is not bounded $\left(\left|C_{n}\right| \rightarrow+\infty\right)$ we have that

$$
\left\|\frac{u_{n}}{\sqrt{\left|C_{n}\right|}}-\frac{C_{n}}{\sqrt{\left|C_{n}\right|}}\right\|_{H^{1}}^{2} \leq \frac{A}{\left|C_{n}\right|}+B \quad \forall n \geq n_{0}
$$

Hence, it is bounded, so there exists $u \in H^{1}$ and a subsequence $n_{j}$ such that $\frac{u_{n_{j}}}{\sqrt{\left|C_{n_{j}}\right|}}-\frac{C_{n_{j}}}{\sqrt{\left|C_{n_{j}}\right|}} \rightarrow u$ weakly in $H^{1}$

We could also say that for almost all $x$ we have

$$
u_{n_{j}}(x) \sim u(x) \sqrt{\left|C_{n_{j}}\right|}+C_{n_{j}}
$$

In particular this says that $\left|u_{n_{j}}\right| \rightarrow+\infty$. Note that we can write it as:

$$
u_{n_{j}}=\frac{u_{n_{j}}}{\left|u_{n_{j}}\right|}\left|u_{n_{j}}\right|
$$

As $\frac{u_{n_{j}}}{\left|u_{n_{j}}\right|} \in S^{N-1}$, by hypothesis $\left(P_{1}\right)$, there exists $U_{k} \subset S^{N-1}$ with $\frac{u_{n_{j}}}{\left|u_{n_{j}}\right|} \in U_{k}$ and a $w_{k} \in S^{N-1}$ such that

$$
\left\langle g\left(\frac{u_{n_{j}}}{\left|u_{n} j\right|} R\right), w_{k}\right\rangle<0
$$

for $R$ big enough, so as $\left|u_{n_{j}}\right| \rightarrow \infty$, we have that for $n_{j}$ sufficiently large: 


$$
\left\langle g\left(u_{n_{j}}\right), w_{k}\right\rangle<0
$$

But we had that $\int_{\Omega} g\left(u_{n_{j}}\right) d x=0$. These two last statements lead to a contradiction. Hence $\left|C_{n_{j}}\right|$ is bounded, and the result follows.

Lemma 3.5 There exists $R>0$ such that $\left\|u_{n}\right\|_{\infty} \leq R$ for all $n \in \mathbb{N}$.

Proof:

The adapted Stampacchia result, both for a system and for this type of boundary conditions, says that for each perturbed problem, if $u_{n}$ is a solution in $L^{p}$, then it is bounded in $L^{\infty}$ by the following:

$$
\left\|u_{n}\right\|_{\infty} \leq\left|C_{n}\right|+K\left\|f-g_{n}\left(u_{n}\right)\right\|_{L^{p}}|\Omega|^{\frac{1}{d}-1 p} \mathcal{C}
$$

As we already seen that the $C_{n}$ are uniformly bounded, and also the $L^{p}$ norm of $f-g_{n}$. Hence, we have the desired uniform bound.

The previous result says that given a sequence $\varepsilon_{n} \rightarrow 0$, we have solution for every $n \in \mathbb{N}$ of problem (2) and that the solution $u_{n} \in C\left(\bar{\Omega}, \mathbb{R}^{N}\right) \cap\{r \leq$ $\left.\left\|u_{n}\right\|_{L^{\infty}} \leq R\right\}$. Regularity Theory tells us that if $f$ is good enough, $u_{n}$ will be a classical solution of our approximated problem.

Proof of Theorem 1.2:

Taking $\varepsilon_{n} \rightarrow 0$, Theorem 3.2 gives, for each $n \in \mathbb{N}$, we consider a solution $u_{n}$ of (2). The lemmas above also garantee that for $n \geq n_{0}$, there exist $r<R$

$$
\tilde{r} \leq\left\|u_{n}\right\|_{L^{\infty}} \leq R
$$

Here is where condition $\left(P_{2}\right)$ is important. Because of the repulsiveness condition, we have that $\operatorname{deg}\left(g_{n}, B_{\tilde{r}}, 0\right)=(-1)^{N}$. Taking $D=\{\tilde{r}<|u|<R\}$, we can verify, because of the esicion propery of the topological degree that

$\operatorname{deg}\left(g, B_{R} \backslash B_{\tilde{r}}, 0\right)=\operatorname{deg}\left(g, B_{R},, 0\right)-\operatorname{deg}\left(g, B_{\tilde{r}}, 0\right)=\operatorname{deg}\left(g, B_{R},, 0\right)-(-1)^{N}$

Here it is also used that if $n$ is big enough, $g_{n} \equiv g$ outside $B_{\tilde{r}}$.

One can also assure that there exists a subsequence of $u_{n}$ (that we still call $u_{n}$ ) and a function $u \in H^{1}$ such that $u_{n} \rightarrow u$ weakly in $H^{1}$. Let us see that this $u$ is in fact a generalized solution of problem (1).

If $u \neq 0$, by definition, as $g_{n} \rightarrow g$ uniformly, $u$ is a solution of the problem

$$
\Delta u+\hat{g}(u)=f \quad \Omega
$$

in the sense of distributions, and as $\left|C_{n}\right|$ are also uniformly bounded, one can assure that $u \in H=H_{0}^{1}+\mathbb{R}^{N}$, and hence, a generalized solution of (1).

If $u=0$ then $\hat{g}(0)$ acts as $f$ and the equation holds. 


\section{Acknowledgments}

We thank Pablo De Nápoli for his ideas on the subject and his appreciations at the genesis of this work.

This work has been supported by Grant UBACyT 20020090100067 from the University of Buenos Aires and CONICET PIP 11220090100637.

\section{References}

[1] P. Amster and P. De Nápoli. Landesman-Lazer type conditions for a system of $p$-Laplacian like operators. J. Math. Anal. Appl., 326(2):1236$1243,2007$.

[2] Pablo Amster and Mónica Clapp. Periodic solutions of resonant systems with rapidly rotating nonlinearities. Discrete Contin. Dyn. Syst., 31(2):-, 2011.

[3] Pablo Amster and Manuel Maurette. Periodic solutions of systems with singularities of repulsive type. Adv. Nonlinear Stud., 11(1):201-220, 2011.

[4] David Arcoya, Sara Barile, and Pedro J. Martínez-Aparicio. Singular quasilinear equations with quadratic growth in the gradient without sign condition. J. Math. Anal. Appl., 350(1):401-408, 2009.

[5] Henri Berestycki and Haïm Brézis. On a free boundary problem arising in plasma physics. Nonlinear Anal., 4(3):415-436, 1980.

[6] Lucio Boccardo and Luigi Orsina. Semilinear elliptic equations with singular nonlinearities. Calc. Var. Partial Differential Equations, 37(34):363-380, 2010.

[7] V. Coti Zelati. Dynamical systems with effective-like potentials. Nonlinear Anal., 12(2):209-222, 1988.

[8] A. Fonda and R. Toader. Periodic orbits of radially symmetric Keplerian-like systems: a topological degree approach. J. Differential Equations, 244(12):3235-3264, 2008.

[9] J. Mawhin. Topological degree methods in nonlinear boundary value problems, volume 40 of CBMS Regional Conference Series in Mathematics. American Mathematical Society, Providence, R.I., 1979. Expository lectures from the CBMS Regional Conference held at Harvey Mudd College, Claremont, Calif., June 9-15, 1977. 
[10] L. Nirenberg. Generalized degree and nonlinear problems. In Contributions to nonlinear functional analysis (Proc. Sympos., Math. Res. Center, Univ. Wisconsin, Madison, Wis., 1971), pages 1-9. Academic Press, New York, 1971.

[11] Rafael Ortega. Nonexistence of radial solutions of two elliptic boundary value problems. Proc. Roy. Soc. Edinburgh Sect. A, 114(1-2):27-31, 1990.

[12] D. Ruiz and J. R. Ward Jr. Some notes on periodic systems with linear part at resonance. Discrete Contin. Dyn. Syst., 11(2\& 3):337-350, 2004.

[13] S. Solimini. On forced dynamical systems with a singularity of repulsive type. Nonlinear Anal., 14(6):489-500, 1990.

[14] Guido Stampacchia. Le problème de Dirichlet pour les équations elliptiques du second ordre à coefficients discontinus. Ann. Inst. Fourier (Grenoble), 15(fasc. 1):189-258, 1965.

[15] Guido Stampacchia. Èquations elliptiques du second ordre à coefficients discontinus. Séminaire de Mathématiques Supérieures, No. 16 (Été, 1965). Les Presses de l'Université de Montréal, Montreal, Que., 1966.

[16] R. Temam. A non-linear eigenvalue problem: the shape at equilibrium of a confined plasma. Arch. Rational Mech. Anal., 60(1):51-73, 1975/76. 